Mantener la "sana distancia" es una estrategia efectiva para reducir la probabilidad del contagio del SARS-COV-2. Sin embargo, el confinamiento implica un costo biopsicosocial muy alto, pues se puede convertir en una situación estresante $y$, sin certidumbre de cuándo este terminará, podría generar que las personas entraran en una fase de estrés crónico, el cual altera el funcionamiento de los sistemas nervioso autónomo, endócrino e inmunológico. Para evaluar el funcionamiento emocional y social individual se puede utilizar la imagen térmica infrarroja, la cual examina los cambios de temperatura faciales y en manos (respuesta del sistema nervioso autónomo) para enfrentar las demandas ambientales, dando lugar a la termorregulación afectiva. Se recomiendan algunas acciones a seguir para regular la actividad nerviosa autonómica a la distancia social

Palabras clave: termorregulación afectiva, estrés social, apoyo social, temperatura nasal

\title{
Los efectosidel estrés social en tiempos de mantener la sana distancia: termorregulación afectiva
}

\section{El aislamiento social que promueve la "sana distancia"}

En semanas pasadas se han publicado un sinnúmero de notas periodísticas académicas y del Gobierno federal sobre la importancia de mantener una "sana distancia" como medida preventiva de contagio del sars-Cov-2. Dos ejemplos de lo anterior se publicaron en el portal de la Comisión Universitaria para la Atención de la Emergencia Coronavirus (2020a, 2020b) de la UNAM, donde se aborda el distanciamiento social como "barrera efectiva para impedir la transmisión del coronavirus", y que este "puede haber salvado ocho millones de vidas en el mundo". La relevancia de esta medida preventiva es inobjetable, pero con un costo biopsicosocial muy alto.

El aislamiento social se define como un déficit en la interacción social humana asociada a efectos negativos para la salud:

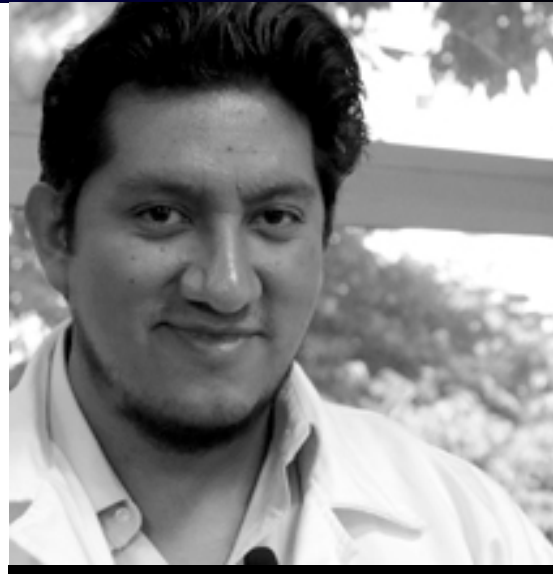

Investigador posdoctoral del CRIM 


\section{ca}

Se ha promovido el uso de la comunicación

a distancia, como las videollamadas, videoconferencias

y clases virtuales. Su efectividad está por evaluarse

al término del confinamiento total por CovID-19."

compromete el funcionamiento cognitivo y del sistema cardiovascular y neuroendocrino; además, puede propiciar depresión y trastornos de sueño (Bhatti y Haq, 2017).

Para acotar el efecto del aislamiento social, se ha promovido el uso de la comunicación a distancia, como las videollamadas, videoconferencias y clases virtuales. Su efectividad está por evaluarse al término del confinamiento total por covid-19. Sin embargo, podemos adelantar que el costo de la pérdida de la interacción humana física induce un estado de estrés, primero agudo, y dependiendo de la duración de la limitación del contacto físico, estrés crónico. Bajo una situación amenazante, el organismo presenta una oleada de calor en la frente, con las manos y nariz fría (figura 1). Esta diferencia de temperatura facial es una manifestación del sistema nervioso autónomo cuando interactúa con demandas ambientales: mientras mayor es el esfuerzo cognitivo, mayor es la diferencia entre la temperatura nasal y la temperatura frontal (Abdelrahman, Velloso, Dingler, Schmidt y Vetere, 2017).

\section{Figura 1}

Imagen térmica infrarroja de una persona con estrés y ansiedad

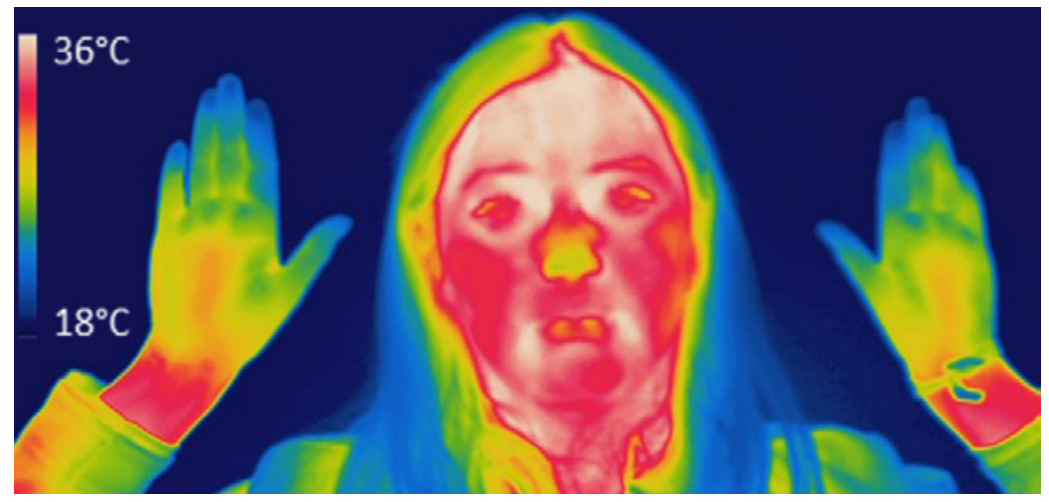

Fuente: Autor (Rodríguez-Medina, Domínguez, Leija, Ortíz y Carreño, 2018).

\section{¿Contar con un equipo con conexión a internet asegura la conexión social?}

Mucho antes de la pandemia de covid-19, contar con la posibilidad de tener a nuestro alcance un equipo con conexión a internet no garantizaba sentirnos acompañados. En muchos casos, una persona puede sentirse sola aun cuando cohabite y/o trabaje con otras personas, con o sin acceso a internet.

Si revisamos las cifras de salud mental, notaremos que existe un importante sector de la población en México con problemas de ansiedad, depresión y otras psicopatologías. Es posible que la sana distancia las haya exacerbado. Para mitigar el problema, el gobierno federal y algunos gobiernos locales, así como las instituciones de educación superior públicas, han puesto sus servicios de asistencia psicológica al alcance de la población utilizando los medios virtuales y telefónicos. Recientemente el Gobierno federal, junto con un grupo de especialistas de la UNAM, publicaron un instrumento en línea sobre los síntomas 
emocionales para la detección de riesgos a la salud mental (ssa y UNAM, 2020). En una pregunta de este cuestionario se indaga con quién convive la persona (familiares, amigos o nadie). Sin embargo, es insuficiente para conocer el nivel de apoyo social que una persona percibe, ya que las preguntas apropiadas para medirlo deben ser si el participante

ca

La cultura mexicana promueve

las prácticas sociales de contacto físico

entre familiares, amistades y relaciones interpersonales

con muestras de aprecio que alientan

la cercanía afectiva y retroalimentan

la calidad de la relación afectiva." cuenta con el apoyo instrumental y afectivo de familiares, amigos, o se siente solo, con qué frecuencia y en qué medida. La falta de apoyo social induce un sentimiento de desconfianza y un estado de intranquilidad que se manifiestan en nuestro organismo con la participación del sistema nervioso autónomo de defensa (Bhatti y Haq, 2017). Y al contrario, cuando nos sentimos apoyados por nuestro grupo, como la familia, amigos, compañeros o la pareja, es más probable que nos encontremos relajados, receptivos, y que esta calma tienda manifestarse en la temperatura facial y en manos.

\section{Termorregulación afectiva}

Nuestro sistema nervioso, como el de la mayoría de los mamíferos, está programado para tener contacto social. La cultura mexicana promueve prácticas sociales de contacto físico entre familiares, amistades, y relaciones interpersonales con muestras de aprecio que alientan la cercanía afectiva y retroalimentan la calidad de la relación afectiva. El calor de un abrazo o un beso estimula los termorreceptores (no nocivos), que son procesados en el sistema nervioso por estructuras subcorticales que dan lugar a una sensación agradable, lo que aumenta la temperatura nasal (Gómez y Moliné, 2016). Si confiamos en la persona, se percibe un apoyo social afectivo.

La interacción social promueve la actividad del sistema nervioso autónomo: en una situación inesperada, se activa nuestro sistema simpático. Ya que en una situación desesperada, como el confinamiento que llevamos para no enfermar de covid-19, la actividad simpática permanece elevada, lo que implica otras manifestaciones inmunológicas, como la respuesta inflamatoria. En contraparte, cuando nos involucramos socialmente predomina la actividad del sistema nervioso parasimpático, la cual nos facilita sentirnos en confianza. La falta de contacto físico con las personas en quienes confiamos para no contagiarnos puede incrementar la actividad simpática si no contamos con otras fuentes de serenidad en el hogar, como los pasatiempos, la lectura, el desarrollo creativo o la comunicación emocional (positiva y negativa).

\section{Diagnóstico e intervención biopsicosocial en personas con a situaciones sociales estresantes}

El estrés, como respuesta fisiológica, puede ser evaluado mediante la imagen térmica infrarroja, al igual que la respuesta fisiológica de relajación. La punta de la nariz es de particular interés para la investigación psicofisiológica social: ante un estresor, físico o psicológico, la temperatura nasal tiende a disminuir; en cambio, cuando estamos tranquilos, la tempe- 
ratura nasal tiende a aumentar (Rodríguez-Medina, Domínguez, Ortíz, Leija y Chavarría, 2018b). Esta respuesta térmica corporal puede considerarse un indicador objetivo de la capacidad de regulación emocional.

El confinamiento y el distanciamiento social por riesgo de enfermar de covid-19 pueden precipitar la respuesta de estrés, manifestándose con temperatura nasal baja, lo cual puede influir en la percepción de apoyo social (Bhatti y Haq, 2017) y el estado anímico, y debilitar el sistema inmunológico, aumentando el riesgo de proliferación de virus y la actividad inflamatoria (Rodríguez-Medina et al., 2019). Por el contrario, los agentes protectores, como el apoyo social, la expresión y regulación emocional, así como las actividades agradables, pueden aumentar la temperatura y mantener la respuesta inmune.

Hasta el momento, los datos recolectados para la evaluación del funcionamiento autonómico en una situación psicosocial, en un grupo de jóvenes universitarios y un grupo de adultos mayores, sugieren una asociación moderada entre la temperatura nasal y el puntaje de apoyo social (figura 2). El objetivo principal de esta investigación es estudiar los mecanismos de termorregulación afectiva en situaciones sociales estresantes, cuya aplicación sería distinguir entre aquellas personas que requieren apoyo psicológico especializado, y quienes no lo necesitan.

Figura 2

Asociación entre la temperatura nasal y el apoyo social

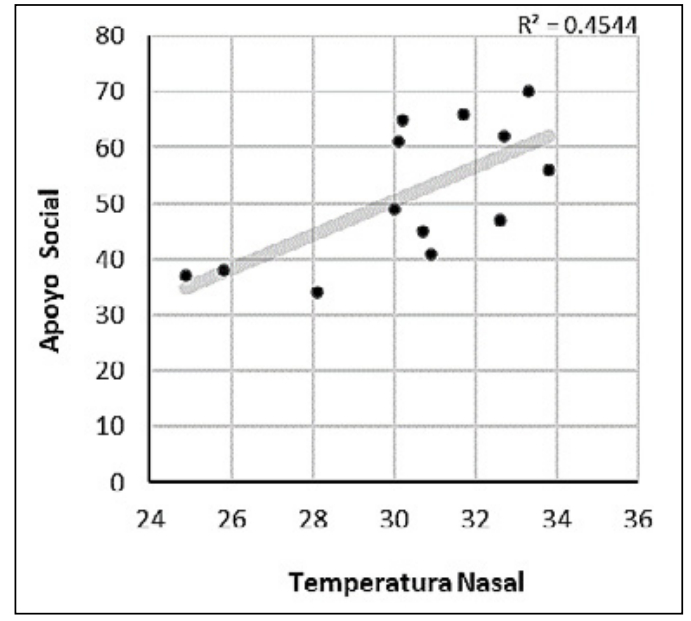

Fuente: Autor.

La figura 2 presenta la relación entre la temperatura nasal y el apoyo social. Las personas con menor percepción de apoyo social son aquellas que están más frías (estresadas).

\section{Recomendaciones para la regulación térmica agradable durante el confinamiento por covid-19}

Además de seguir las indicaciones oficiales de las autoridades sanitarias para promover el bienestar psicológico, se pueden practicar al menos dos o más actividades de manera permanente cuando no contamos con la posibilidad del contacto social habitual:

- La respiración diafragmática 
- La meditación

- Terapias mente-cuerpo: yoga, tai- chi

- Ejercicio físico cardiovascular

- Actividades cognitivas estimulantes de la atención: leer, jugar, programar planes agradables

- Actividades físicas que le resulten agradable a la persona: cantar y/o tocar un instrumento musical, bailar, cocinar, armar, reorganizar el espacio físico.

- Actividades de interacción social que le resulten placenteras a la persona: primero, sostener conversaciones por videollamada o llamadas telefónicas; segundo, comunicarse por correo, priorizando sus necesidades y sentimientos; tercero, el uso de las redes sociales virtuales con temas de interés común, sin abusar de estas redes por más de 60 minutos al día, pues la persona puede dedicarle más tiempo a esta actividad pasiva, con los riesgos asociados al sedentarismo

\section{ca} La falta de contacto físico con las personas en quienes confiamos para no contagiarnos puede incrementar la actividad simpática si no contamos con otras fuentes de serenidad en casa, como los pasatiempos,

la lectura, el desarrollo creativo o la comunidación emocional."
Estas actividades (económicas y no invasivas) van a promover la actividad nerviosa simpática y su regulación autonómica parasimpática, la cual inducirá cambios térmicos que pueden percibirse como agradables. Estas prácticas no pretenden sustituir el contacto social, suspendido temporalmente, sino manejar, con su incorporación, el estrés por el confinamiento de manera individual e incluso familiar dentro del hogar.

\section{Referencias bibliográficas}

Abdelrahman, Y., Velloso, E., Dingler, T., Schmidt, A. y Vetere, F. (2017). Cognitive heat. Proceedings of the ACM on Interactive, Mobile, Wearable and Ubiquitous Technologies, 1(3), 1-20. https://doi.org/10.1145/3130898

Bhatti, A. y Haq, A. (2017). The pathophysiology of perceived social isolation: Effects on health and mortality. Cureus, 9(1): e994. https://doi.org/10.7759/cureus.994

Comisión Universitaria de Atención a la Emergencia Coronavirus (25 de abril de 2020a). El distanciamiento social puede haber salvado ocho millones de vidas en el mundo. México: unAm. https://covid19comisionunam.unamglobal.com/?p=84838\&fbclid =IwAR36tjCw06F7GsMuMtHXJzj-uIxexmEYHJHATgykDfWph1ZDxDkiH FyjUtg

Comisión Universitaria de Atención a la Emergencia Coronavirus (23 de marzo de 2020b). Distanciamiento social, barrera efectiva para impedir la transmisión del coronavirus. México: UNAM. https://covid19comisionunam.unamglobal.com/?p=83414

Gómez, E. y Moliné, A. (10 de octubre de 2016). El calor de un beso. Revista QUO. https:// www.researchgate.net/publication/318405219_El_calor_de_un_beso

Rodríguez-Medina, D., Domínguez, B., Leija, G., Ortiz, M. y Carreño, C. (2018a). Efectos psicofisiológicos de la respiración diafragmática y la estimulación térmica sobre la actividad autonómica del estrés agudo. Acta de Investigación Psicológica, 8(2), 101-113. http://dx.doi.org/10.22201/fpsi.20074719e.2018.2.09

Rodríguez-Medina, D., Domínguez, B., Ortiz, M., Leija, G. y Chavarría, O. (2018b). 
Efecto de la valencia afectiva del pensamiento sobre la temperatura nasal: imaginería guiada y estrés psicosocial. Psicología y Salud, 28(2), 187-194. https:// doi.org/10.25009/pys.v28i2.2555

Rodríguez-Medina, D., Leija-Alva, G., Domínguez-Trejo, B., Hernández-Pozo, M., Cruz-Albarrán, I., Morales-Hernández, L., \& Marmolejo-Ramos, F. (2019). Effects of the Trier Social Stress Test on the distributions of IL-6 and MAP levels. Heliyon, 5(4), e01580. https://doi.org/10.1016/j.heliyon.2019.e01580

ssa y unam (Secretaría de Salud y Universidad Nacional Autónoma de México) (2020). Cuestionario para la detección de riesgos a la salud mental. México: Dirección General de Atención a la Comunidad-unam; Facultad de Psicología-unam; Instituto Nacional de Psiquiatría Ramón de la Fuente Muñiz. https://misalud.unam.mx/covid19/?fbclid=IwAR31DSNys48yOduypufC99ythLZgphAI2En1Z26TVxomMjlrhmry75seCK0 